\title{
Color-flavor locked strangelets
}

\author{
Jes Madsen \\ Institute of Physics and Astronomy, University of Aarhus, DK-8000 Arhus C, Denmark
}

(August 3, 2001; Published in Phys.Rev.Lett. 87 (2001) 172003)

Finite lumps of color-flavor locked strange quark matter (CFL-strangelets) are significantly more stable than strangelets without color-flavor locking for wide ranges of parameters, increasing the likelihood of strangelet metastability, or even absolute stability beyond some minimum baryon number $A_{\min }$. Whereas bulk CFL strange quark matter is electrically neutral, CFL-strangelets are positively charged, with $Z \approx 0.3 A^{2 / 3}$. This is quite different from "ordinary" strangelets and may provide a possible test of color-flavor locking if strangelets are detected in upcoming cosmic-ray space experiments.

12.38.Mh, 12.39.Ba, 24.85.+p, 96.40.-z

It has recently been demonstrated that quark matter at high density may be in a so-called color-flavor locked phase where quarks with different color and flavor quantum numbers form Cooper pairs with very large binding energy [1]. Such a state is significantly more bound than ordinary quark matter, and this increases the likelihood that quark matter composed of up, down, and strange quarks may be metastable or even absolutely stable. In other words color-flavor locked quark matter rather than nuclear matter may be the ground state of hadronic matter. The present Letter discusses this possibility with particular emphasis on the consequences for finite size quark matter lumps, so-called strangelets.

As shown by Rajagopal and Wilczek [2] the color-flavor locked phase is electrically neutral in bulk for a significant range of chemical potentials and s-quark mass. The reason for the neutrality is that BCS-like pairing minimises the energy if the quark Fermi-momenta are equal (since pairing happens between quarks of different color and flavor, and opposite momenta $\vec{p}$ and $-\vec{p}$ ). For equal Fermi-momenta, the number of up, down and strange quarks are equal, and the net charge of the system is zero without any need for (or even room for) electrons. This has important consequences for the physics of compact stars containing color-flavor locked quark matter [3]. As demonstrated below, it also has important consequences for the mass and charge properties of finite lumps of strange quark matter (strangelets) if these consist of color-flavor locked rather than "normal" quark matter.

For "normal" quark matter it is known that finite size effects increase the energy 国 14, and make the charge more positive 14. As shown below a similar thing happens in color-flavor locked quark matter. Even for quark matter where equal Fermi momenta are enforced by color-flavor locking, the finite-size effects change the net quark charge of the system from zero to a positive value. The resulting charge-mass relation $\left(Z \approx 0.3 A^{2 / 3}\right)$ differs significantly from ordinary strangelets and may allow for an experimental test of color-flavor locking.

Following Refs. [2, 3, the color-flavor locked phase with full pairing has a free energy (volume terms only)

$\Omega_{\mathrm{CFL}, V} V=\Omega_{\mathrm{free}, V}\left(\mu_{u}, \mu_{d}, \mu_{s}, p_{F u}, p_{F d}, p_{F s}\right) V+\Omega_{\mathrm{pair}, V} V$

where $\Omega_{\text {pair }, V} \approx-3 \Delta^{2} \mu^{2} / \pi^{2}$, with $\Delta \approx 10-100 \mathrm{MeV}$ being the pairing energy gap and $\mu$ the average quark chemical potential. $\Omega_{\mathrm{free}, V}$ is the usual Fermi-gas result (see below) as a function of the individual quark chemical potentials, $\mu_{i}$, and Fermi-momenta, $p_{F i}$, but for the color-flavor locked phase the Fermi-momenta are all equal, $p_{F u}=p_{F d}=p_{F s} \equiv p_{F}$ [2].

Finite lumps of quark matter have additional contributions to $\Omega_{\text {free }}$ in terms of surface and curvature energies. Presumably, also $\Omega_{\text {pair }}$ would contain finite-size terms, but these are likely to be small compared to the corrections to $\Omega_{\text {free }}$, as long as $\Omega_{\text {pair }}$ itself is a perturbation to $\Omega_{\text {free }}$. Here such higher order terms will be neglected, and it will be assumed that the CFL state keeps all quark Fermi momenta equal to optimize the pairing energy. The common value of $p_{F}$ is found by minimizing $\Omega_{\text {free }}$ at fixed radius, and the equilibrium radius is found by minimizing the total energy with respect to radius at fixed baryon number.

The calculation of the unpaired contributions, $E$, to the total energy, $E_{\mathrm{CFL}}=E+\Omega_{\text {pair }}$, is performed within the MIT bag model [15], with $\alpha_{S}=0$. Here the energy of a system composed of quark flavors $i$ is given by $E=\sum_{i}\left(\Omega_{i}+N_{i} \mu_{i}\right)+B V$, where $\Omega_{i}, N_{i}$ and $\mu_{i}$ denote thermodynamic potentials, total number of quarks, and chemical potentials, respectively. $B$ is the bag constant, $V$ is the bag volume. The thermodynamical quantities can be derived from a density of states of the form [16] $\frac{d N_{i}}{d k}=6\left\{\frac{k^{2} V}{2 \pi^{2}}+f_{S}\left(\frac{m_{i}}{k}\right) k S+f_{C}\left(\frac{m_{i}}{k}\right) C+\ldots\right\}$, where a sphere has area $S=4 \pi R^{2}$ and curvature $C=8 \pi R$. The functions $f_{S}$ and $f_{C}$ depend on the boundary conditions. For the MIT-bag model $f_{S}(m / k)=-\left[1-(2 / \pi) \tan ^{-1}(k / m)\right] / 8 \pi[8]$ and $f_{C}(m / k)=\left[1-3 k /(2 m)\left(\pi / 2-\tan ^{-1}(k / m)\right)\right] / 12 \pi^{2}$ [12]. The number of quarks of flavor $i$ is $N_{i}=$ $\int_{0}^{p F i}\left(d N_{i} / d k\right) d k=n_{i, V} V+n_{i, S} S+n_{i, C} C$, and the corresponding thermodynamic potentials are $\Omega_{i}=$ $\int_{0}^{p_{F i}}\left(d N_{i} / d k\right)\left(\epsilon_{i}(k)-\mu_{i}\right) d k=\Omega_{i, V} V+\Omega_{i, S} S+\Omega_{i, C} C$, 
where $\epsilon_{i}(k)=\left(k^{2}+m_{i}^{2}\right)^{1 / 2}$. The expressions obey $\partial \Omega_{i} / \partial \mu_{i}=-N_{i}$, and $\partial \Omega_{i, j} / \partial \mu_{i}=-n_{i, j}$.

With $\lambda_{i} \equiv m_{i} / p_{F i}$ this gives

$$
\begin{aligned}
& \Omega_{i, V}=-\frac{3 p_{F i}^{4}}{8 \pi^{2}}\left(\frac{8 \mu_{i}}{3 p_{F i}}-2\left(1+\lambda_{i}^{2}\right)^{3 / 2}+\lambda_{i}^{2}\left(1+\lambda_{i}^{2}\right)^{1 / 2}\right. \\
& \left.+\lambda_{i}^{4} \ln \frac{1+\left(1+\lambda_{i}^{2}\right)^{1 / 2}}{\lambda_{i}}\right)
\end{aligned}
$$

and

$$
n_{i, V}=p_{F i}^{3} / \pi^{2} .
$$

Massless quarks have $\Omega_{i, S}=0$, with a significantly more cumbersome equation for s-quarks. The corresponding change in quark number per unit area,

$$
n_{i, S}=-\frac{3}{4 \pi} p_{F i}^{2}\left[\frac{1}{2}+\frac{\lambda_{i}}{\pi}-\frac{1}{\pi}\left(1+\lambda_{i}^{2}\right) \tan ^{-1}\left(\lambda_{i}^{-1}\right)\right],
$$

is always negative, approaching 0 for $\lambda_{i} \rightarrow 0$ (massless quarks) and $-3 p_{F i}^{2} / 8 \pi$ for $\lambda_{i} \rightarrow \infty$.

For massless $\mathrm{u}$ and $\mathrm{d}$ quarks $\Omega_{i, C}=\left(2 p_{F i} \mu_{i}-\right.$ $\left.p_{F i}^{2}\right) /\left(8 \pi^{2}\right)$, and $n_{i, C}=-p_{F i} /\left(4 \pi^{2}\right)$. For a massive quark

$$
n_{i, C}=\frac{p_{F i}}{8 \pi^{2}}\left[1-\frac{3 \pi}{2 \lambda_{i}}+3 \frac{1+\lambda_{i}^{2}}{\lambda_{i}} \tan ^{-1}\left(\lambda_{i}^{-1}\right)\right] .
$$

Quark matter in weak equilibrium has $\mu_{s}=\mu_{d}=\mu_{u}+$ $\mu_{e}$, maintained by reactions like $u+d \leftrightarrow s+u, u+$ $e^{-} \leftrightarrow d+\nu_{e}$. For a system with zero electron chemical potential, e.g. a finite strangelet with $A \ll 10^{7}$ (for $A>$ $10^{7}$ the strangelet radius exceeds the electron Compton wavelength, so that some electrons may be trapped inside the quark phase), weak interactions keep an equilibrium characterised by $\mu_{u}=\mu_{d}=\mu_{s} \equiv \mu$.

The surface and curvature terms have two important implications for the color-flavor locked strangelets. First, as for normal strangelets, they destabilize small systems relative to bulk quark matter. Second and perhaps more important, quark lumps in the color-flavor locked phase with equal Fermi-momenta are no longer electrically neutral as in bulk, but instead gain a net positive quark charge due to the relative suppression of s-quarks for fixed $p_{F}$, similar to the charge increase which has been pointed out for normal quark matter 14.

In bulk, equal Fermi-momenta means equal quark numbers since the pairing terms are equal [2], $N_{i \text {,pair }}=$ $V 2 \Delta^{2} \mu / \pi^{2}$, and the contribution from $\Omega_{\text {free }}$ is $N_{i \text {,free }}=$ $V p_{F}^{3} / \pi^{2}$. But for finite systems, the number of massive quarks is generally reduced relative to massless quarks at fixed $p_{F}$. This is explicitly the case for the MIT bag model boundary conditions, where surface tension is related to a suppression of the s-quark wave-function at the surface. MIT bag boundary conditions correspond to no flux of quarks across the surface. For a massive s-quark this lowers the s-quark density near the surface relative to that of $\mathrm{u}$ and $\mathrm{d}\left(n_{s, S}<0\right)$, leading to a net increase in the total electrical charge, which therefore must become positive for quark matter with equal Fermimomenta, i.e. color-flavor locked quark matter. This is a consequence of quantum mechanics with validity beyond the MIT bag model. In the nonrelativistic limit of a very massive quark the wave function must be zero at the boundary. This is not the case for a relativistic quark. Therefore one would generally expect massive s quarks to be more suppressed than $\mathrm{u}$ and $\mathrm{d}$.

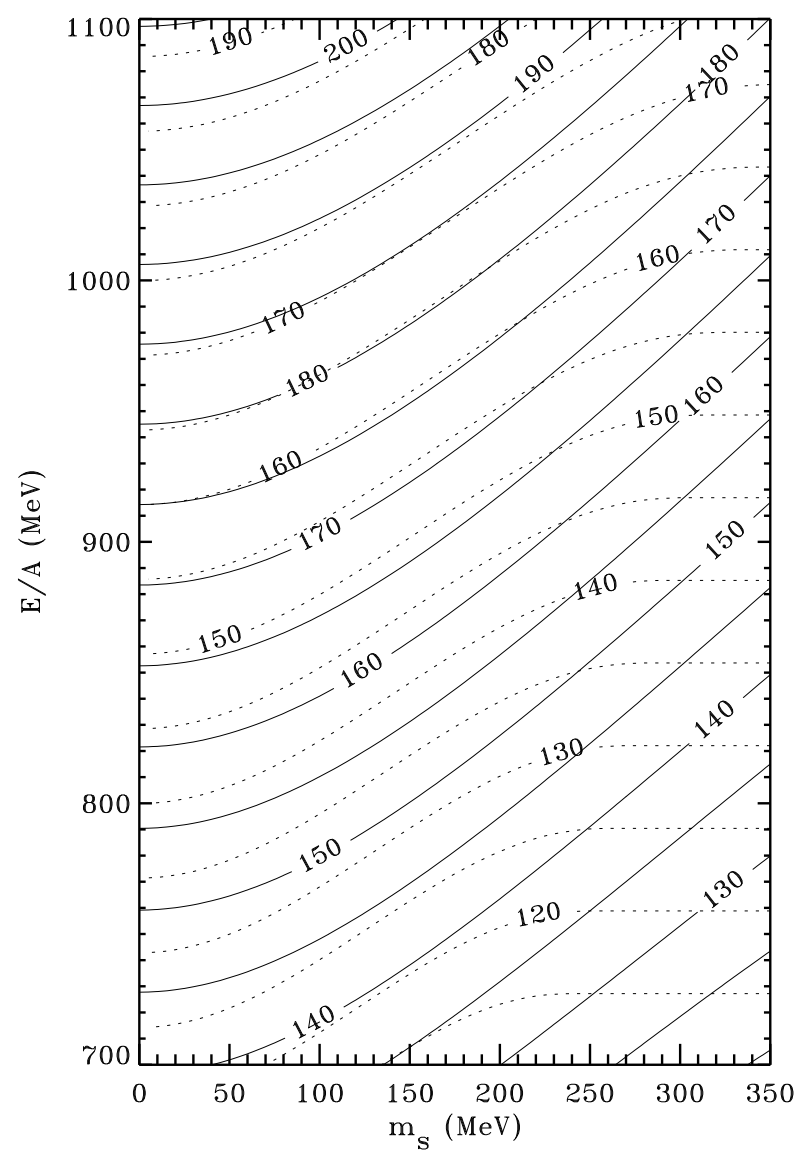

FIG. 1. Energy per baryon in $\mathrm{MeV}$ as a function of $m_{s}$ for bulk strange quark matter with $B^{1 / 4}$ in $\mathrm{MeV}$ as indicated. Full lines are results for color-flavor locked quark matter $(\Delta=100 \mathrm{MeV})$, dashed lines without color-flavor locking.

Figure 1 shows energy per baryon for bulk CFL-quark matter with $\Delta=100 \mathrm{MeV}$ as a function of bag constant and s-quark mass. Results for non-CFL quark matter are shown for comparison. As demonstrated previously (e.g. [1]3]) it is evident that color-flavor locking significantly lowers the energy and makes quark matter more stable. Up to a hundred $\mathrm{MeV}$ per baryon is gained for some parameter choices. This means that strangelets, if they are color-flavor locked, may be absolutely stable $(E / A<930 \mathrm{MeV})$ for bag constants $B^{1 / 4}$ as high as $180 \mathrm{MeV}$ for low strange quark mass, $170 \mathrm{MeV}$ for 
$m_{s} \approx 150 \mathrm{MeV}$, and $160 \mathrm{MeV}$ for $m_{s} \approx 300 \mathrm{MeV}$, whereas stability without color-flavor locking requires $B^{1 / 4}<163 \mathrm{MeV}$ even for massless s-quarks. The corresponding ranges for metastability are pushed to higher $B$ as well. For normal quark matter $B^{1 / 4}$ is bounded from below by the value $146 \mathrm{MeV}$, below which ordinary nuclei would be unstable against spontaneous decay into two-flavor up-down quark matter. For $\Delta=100 \mathrm{MeV}$ this bound increases to $B^{1 / 4}>156 \mathrm{MeV}$ to avoid spontaneous nuclear decay into a two-flavor color superconducting state where two of the three colors of up and down quarks form Cooper pairs [17]. The proper value of $B$ is an important but unsettled issue. The original MIT bag model fits to hadrons implied $B^{1 / 4}=145 \mathrm{MeV}$, but this depends significantly on the choice of $m_{s}, \alpha_{s}$, and not least a phenomenological zero-point energy, which is negligible for larger strangelets. On the other hand, a naive comparison with lattice determinations of the quark-hadron phase transition temperature at $\mu=0$ (circumstances very different from what the bag model was created to describe) would argue for a higher value. Using the bag model result $B^{1 / 4} \approx 1.45 T_{c}$ and the lattice results for $T_{c}$ one finds that $B^{1 / 4}>200 \mathrm{MeV}$. In the latter case, a value of $\Delta$ in excess of the $100 \mathrm{MeV}$ considered here would be necessary for CFL-strangelet stability.

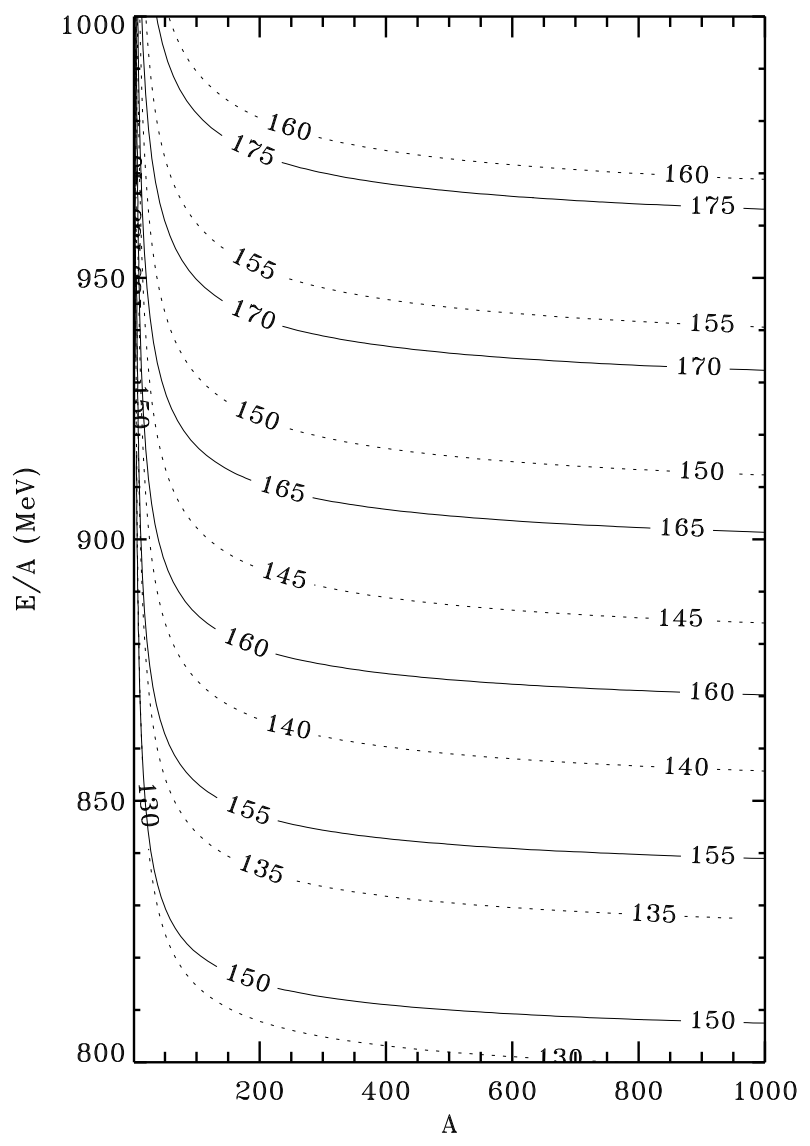

FIG. 2. Energy per baryon in $\mathrm{MeV}$ as a function of $A$ for CFL-strangelets (full curves) and ordinary strangelets (dashed curves) with $B^{1 / 4}$ in $\mathrm{MeV}$ as indicated, $\Delta=100 \mathrm{MeV}$ and $m_{s}=150 \mathrm{MeV}$.

Figure 2 shows energy per baryon for finite size strangelets with and without color-flavor locking. For a given $B$ the strangelet mass-per-baryon increases dramatically for low $A$ because of the surface and curvature energies, and there is a lower bound $A_{\min }$ for the baryon number of stable (metastable), strangelets, which depends on parameters. The overall gain in stability for CFL-strangelets relative to non-CFL strangelets mainly reflects the gain in bulk binding.

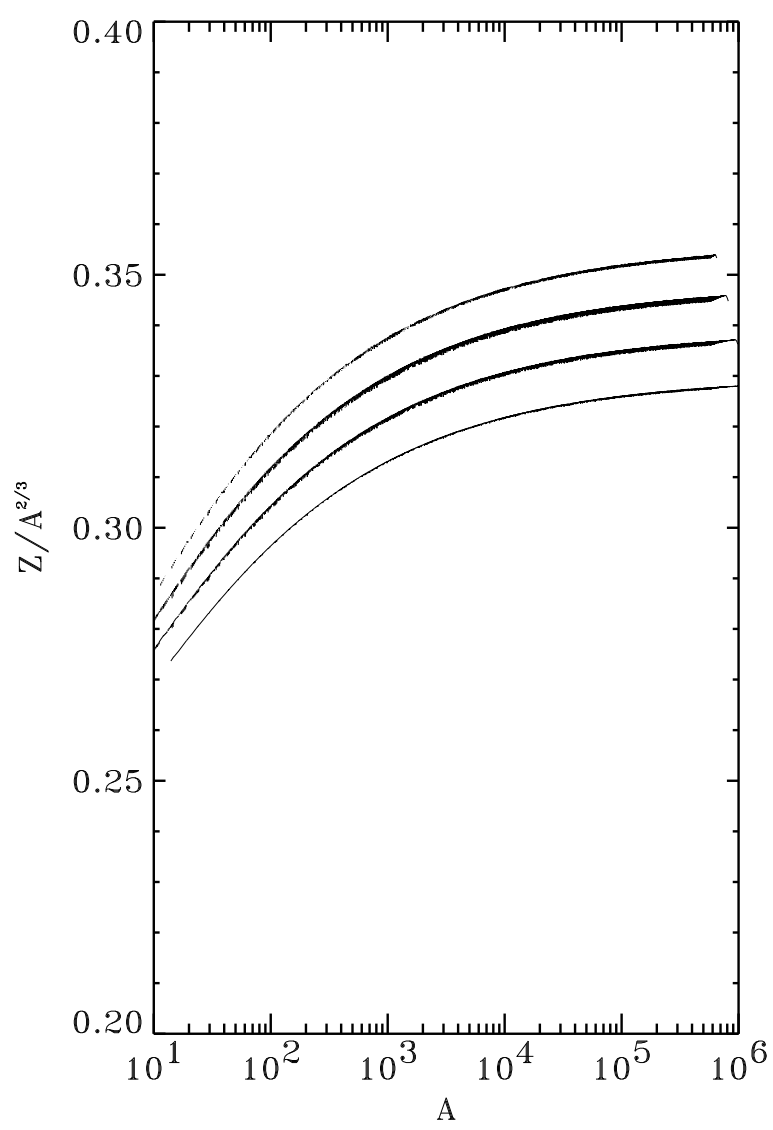

FIG. 3. Charge divided by $A^{2 / 3}$ as a function of $A$ for CFL-strangelets with $B^{1 / 4}=150,160,170$, and $180 \mathrm{MeV}$ (top to bottom), $\Delta=100 \mathrm{MeV}$ and $m_{s}=150 \mathrm{MeV}$.

Perhaps more interesting, and in significant contrast to the properties of non-CFL strangelets, is the charge $Z$ of strangelets as a function of $A$. Since bulk CFL strange quark matter is electrically neutral [2,3], the total charge in the case of finite strangelets with $\mu_{e}=0$ comes from the surface suppression of massive relative to massless quarks following from the MIT boundary conditions (and more generally from the fact that $f_{S}$ is neg- 
ative for a massive quark). As can be seen from Fig. 3, the charge per baryon is almost independent of $B$ and it is only weakly dependent on $\Delta$ and $m_{s}$. The characteristic value is $Z \approx 0.3 A^{2 / 3}$, which can be understood as follows. The main charge contribution comes from $n_{s, S}$ as $Z \approx-(1 / 3) n_{i, S} S \approx(2 / \pi)\left(m_{s} / p_{F}\right)\left(p_{F} R\right)^{2}$ (expansion for small $\left.m_{s}\right)$, saturating at $0.5\left(p_{F} R\right)^{2}$ for high $m_{s}$. Neglecting the pairing contribution to $A$, $A \approx V p_{F}^{3} / \pi^{2}=4\left(p_{F} R\right)^{3} / 3 \pi$, so the $Z \propto A^{2 / 3}$ behavior is clear, and the prefactor is not very parameter dependent. For non-CFL strangelets, the charge is the volume charge density times volume for small $A$, and therefore proportional to $A$ itself $(Z \approx 0.1 A$ ), until the system becomes larger than the Debye screening length ( $\approx 5 \mathrm{fm} ; A \approx 150$ ), beyond which the charge is mainly distributed within a Debye length from the surface, and $Z \approx 8 A^{1 / 3}[7,8,18,19$. Charge screening is negligible for CFL-strangelets because the net charge is already concentrated near the surface, as it is enforced by the surface boundary conditions (in spite of the fact, that the quarks share a common Fermi momentum), whereas the central regions remain charge neutral.

The unusual charge properties, in particular the small $Z / A$-ratio, has long been recognized as a crucial signature for experimental identification of strangelets. The results above show that the actual $(A, Z)$ relation is very different in the case of CFL-strangelets $\left(Z / A \propto A^{-1 / 3}\right)$ as compared to ordinary strangelets $(Z / A$ constant for small $A$ and $\propto A^{-2 / 3}$ for large $A$, reaching $Z / A \propto A^{-1 / 3}$ only asymptotically). This is an important distinction which calls for a re-analysis of some of the limits derived in previous experimental strangelet searches [20]. If strangelets are detected in upcoming cosmic ray space experiments (in particular, the Alpha Magnetic Spectrometer [21] and ECCO 22 will be sensitive to strangelets in different charge (mass) regimes), the difference may also allow an experimental test of color-flavor locking in quark matter.

This work was supported in part by the Theoretical Astrophysics Center under the Danish National Research Foundation, and by the Danish Natural Science Research Council.

[1] M. Alford, K. Rajagopal, and F. Wilczek, Phys. Lett. B422, 247 (1998); R. Rapp, T. Schäfer, E. V. Shuryak, and M. Velkovsky, Phys. Rev. Lett. 81, 53 (1998). For extensive reviews and references on color-flavor locking, see M. Alford, Annu. Rev. Nucl. Part. Sci. (2001) (in press) hep-ph/0102047; K. Rajagopal and F. Wilczek in B. L. Ioffe Festschrift, "At the Frontier of Particle Physics/Handbook of QCD", M. Shifman, ed., (World Scientific, 2001) hep-ph/0011333. For review and references on "ordinary" strange quark matter, see J. Mad- sen, Lecture Notes in Physics 516, 162 (1999) astro$\mathrm{ph} / 9809032$.

[2] K. Rajagopal and F. Wilczek, Phys. Rev. Lett. 86, 3492 (2001).

[3] M. Alford, K. Rajagopal, S. Reddy, and F. Wilczek, hepph/0105009 (2001).

[4] A. R. Bodmer, Phys. Rev. D 4, 1601 (1971).

[5] S. A. Chin and A. K. Kerman, Phys. Rev. Lett. 43, 1292 (1979).

[6] E. Witten, Phys. Rev. D 30, 272 (1984).

[7] E. Farhi and R. L. Jaffe, Phys. Rev. D 30, 2379 (1984).

[8] M. S. Berger and R. L. Jaffe, Phys. Rev. C 35, 213 (1987); 44, 566(E) (1991).

[9] J. Madsen, Phys. Rev. Lett. 70, 391 (1993).

[10] E. P. Gilson and R. L. Jaffe, Phys. Rev. Lett. 71, 332 (1993).

[11] J. Madsen, Phys. Rev. D 47, 5156 (1993).

[12] J. Madsen, Phys. Rev. D 50, 3328 (1994).

[13] J. Schaffner-Bielich, C. Greiner, A. Diener, and H. Stöcker, Phys. Rev. C 55, 3038 (1997).

[14] J. Madsen, Phys. Rev. Lett. 85, 4687 (2000).

[15] T. A. DeGrand, R. L. Jaffe, K. Johnson, and J. Kiskis, Phys. Rev. D 12, 2060 (1975).

[16] R. Balian and C. Bloch, Ann. Phys. 60, 401 (1970).

[17] Notice that the introduction of pairing does not just act like a rescaling of $B$ with equal consequences for 2 - and 3 -flavor systems. This is because pairing in a two flavor system involves only $4 / 9$ of the available degrees of freedom in a three flavor system. Thus, the range of allowed $B$-values corresponding to CFL-strangelet stability or metastability is wider than for ordinary strange quark matter.

[18] H. Heiselberg, Phys. Rev. D 48, 1418 (1993).

[19] For high $A$ even "ordinary" strangelets would be dominated by an $A^{2 / 3}$-behavior; c.f. 14.

[20] For recent reviews of strangelet experiments, see R. Klingenberg, J. Phys. G 25, R273 (1999); T. A. Armstrong et al., Phys. Rev. C 63, 054903 (2001).

[21] The AMS homepage is: http://ams.cern.ch.

[22] A description of ECCO can be found at: http://ultraman.berkeley.edu. 